\title{
Association of hypertriglyceridemic waist phenotype with renal function impairment: a cross-sectional study in a population of Chinese adults
}

Yun Qiu ${ }^{1 \dagger}$, Qi Zhao ${ }^{1 \dagger}$, Na Wang ${ }^{1}$, Yuting Yu' ${ }^{1}$, Ruiping Wang ${ }^{2}$, Yue Zhang ${ }^{1}$, Shuheng Cui ${ }^{1}$, Meiying Zhu ${ }^{2}$, Xing Liu', Yonggen Jiang ${ }^{2^{*}}$ and Genming Zhao ${ }^{1^{*}}$

\begin{abstract}
Background: Hypertriglyceridemic waist (HTGW) phenotype has been suggested as a risk factor for chronic kidney disease (CKD). However, there is limited evidence on the relationship of triglyceride waist phenotypes with estimated glomerular filtration rate (eGFR) status and severity. Our aim was to explore the associations of triglyceride waist phenotypes with reduced eGFR and various decreased eGFR stages among Chinese adults.

Methods: A population-based, cross-sectional study was conducted among Chinese participants aged 20-74 years from June 2016 to December 2017 in Shanghai, China. An eGFR value below $60 \mathrm{~mL} / \mathrm{min} / 1.73 \mathrm{~m}^{2}$ was defined as decreased eGFR. HTGW phenotype was defined as triglyceride (TG) $\geq 1.7 \mathrm{mmol} / \mathrm{L}$ and a waist circumference (WC) of $\geq 90 \mathrm{~cm}$ for men and $\geq 80 \mathrm{~cm}$ for women. We examined the association of triglyceride waist phenotypes with decreased eGFR risk using the weighted logistic regression models.

Results: A total of 31,296 adults were included in this study. Compared with normal TG level/normal WC (NTNW) phenotype, normal TG level/enlarged WC (NTGW) and elevated TG level/enlarged WC (HTGW) phenotypes were associated with the increased risk of decreased eGFR. Multivariable-adjusted ORs (95\% Cl) associated with NTGW, elevated TG level/normal WC (HTNW), and HTGW phenotypes were $1.75(1.41-2.18), 1.29$ (0.99-1.68), and 1.99 (1.542.58), respectively. These associations between triglyceride waist phenotypes and decreased eGFR risk remained across almost all the subgroups, including sex, age, BMI, T2DM, and hypertension. HTGW phenotype was consistently positively associated with the risk of mildly and moderately decreased eGFR, but not with severely decreased eGFR risk.

Conclusions: HTGW was consistently associated with the increased risk of decreased eGFR and various decreased eGFR stages, except for severely decreased eGFR. Further prospective studies are warranted to confirm our findings and to investigate the underlying biological mechanisms.
\end{abstract}

Keywords: Chronic kidney disease, Estimated glomerular filtration rate, Hypertriglyceridemic waist phenotype, Renal function decline

\footnotetext{
*Correspondence: 877162034@qq.com; gmzhao@shmu.edu.cn

${ }^{\dagger}$ Yun Qiu and Qi Zhao contributed equally to this work.

${ }^{2}$ Songjiang District Center for Disease Control and Prevention, Shanghai 201600, China

${ }^{1}$ Department of Epidemiology, School of Public Health, Key Laboratory of Public Health Safety of Ministry of Education, Fudan University, Shanghai 200032, China
}

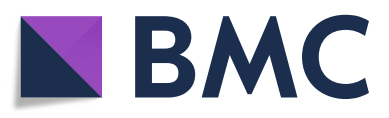

(c) The Author(s). 2020 Open Access This article is licensed under a Creative Commons Attribution 4.0 International License, which permits use, sharing, adaptation, distribution and reproduction in any medium or format, as long as you give appropriate credit to the original author(s) and the source, provide a link to the Creative Commons licence, and indicate if changes were made. The images or other third party material in this article are included in the article's Creative Commons licence, unless indicated otherwise in a credit line to the material. If material is not included in the article's Creative Commons licence and your intended use is not permitted by statutory regulation or exceeds the permitted use, you will need to obtain permission directly from the copyright holder. To view a copy of this licence, visit http://creativecommons.org/licenses/by/4.0/ The Creative Commons Public Domain Dedication waiver (http://creativecommons.org/publicdomain/zero/1.0/) applies to the data made available in this article, unless otherwise stated in a credit line to the data. 


\section{Introduction}

Chronic kidney disease (CKD) remains a main cause of morbidity and mortality worldwide, with increasing prevalence and incidence [1]. One of the characteristics of CKD is a decline of kidney function, which is assessed by estimated glomerular filtration rate (eGFR), a most commonly used marker [2]. The global prevalence of CKD and decreased eGFR $\left(e G F R<60 \mathrm{~mL} / \mathrm{min} / 1.73 \mathrm{~m}^{2}\right)$ in general populations were 14.3 and $9.8 \%$, respectively in 2016 [3]. The overall prevalence of CKD was $10.8 \%$ and decreased eGFR was $1.7 \%$ in the a general population of Chinese adults in 2012 [4]. Recent rapid increases in diabetes, hypertension, and obesity cases will contribute to the increase in CKD prevalence, eventually leading to higher burden of CKD and a bigger threat to public health in less developed regions [5]. Patients with kidney function decline were more likely to have increased risk of cardiovascular events [6], and death from cardiovascular diseases (CVD) [7].

Hypertriglyceridemic waist (HTGW) phenotype is defined by the simultaneous presence of elevated serum triglycerides (TG) level and increased waist circumference (WC). It was first proposed by Lemieux et al. [8], as an indicator of atherosclerosis and an effective tool to identify men who were at high risk of coronary artery disease (CAD). Because assessment of HTGW is relatively inexpensive and easy to acquire, a growing number of studies have shown that HTGW phenotype was associated with the increased risk of CVD [9], CAD [10], hypertension [11], prediabetes [12], and type 2 diabetes mellitus (T2DM) [13], as well as hyperuricemia [14].

Early identification of pertinent risk factors is needed for prevention and control of renal function decline and the development of CKD. In comparison to elevated TG or enlarged WC used alone, the HTGW phenotype is superior in evaluating excess visceral adiposity, and is also a useful clinical tool for identifying individuals with higher risk of abnormal metabolism [15]. Several studies have reported that HTGW phenotype is associated with an increased risk of CKD in adults aged $\geq 40$ years old [16], in elderly (aged $\geq 60$ years old) [17], and in relatively lean people $\left(\mathrm{BMI}<24 \mathrm{~kg} / \mathrm{m}^{2}\right)$ [18]. However, existing evidence regarding the association of HTGW with CKD remains controversial. In a cross-sectional study, Ramezankhani et al. [19] found a positive association of HTGW with CKD only in women; while no significant association between HTGW and CKD was observed in the prospective study. A recent cross-sectional study has demonstrated that HTGW was related to CKD risk in women group but not in men group among adults aged 18 to 75 years old [20]. While another cross-sectional study in elderly participants reached an opposing conclusion [17]. Thus far, limited evidence suggest increased risk of decline renal function with HTGW [21]. Previous studies were limited by the lack of stratified analyses.
Furthermore, no study has reported the relationship of HTGW with the various stages of decreased eGFR, which could represent the progression of CKD.

Using data from physical examinations and electronic medical records, we aimed to examine the association of HTGW and three other triglyceride waist phenotypes with risk of decreased eGFR in overall and subgroup population among Chinese adults. Additionally, we explored whether these phenotypes were associated with different stages of eGFR, including mildly, moderately, and severely decreased eGFR.

\section{Methods}

\section{Study population}

This population-based, cross-sectional study was conducted in Community Health Centers of Songjiang District, Shanghai, China from June 2016 to December 2017. Details of sample methods in this study have been described elsewhere [22]. Briefly, we used a multistage, stratified, clustered sampling method to collect healthrelated data from 31 neighborhood committees and 16 administrative villages in four study community sites, including Zhongshan, Xinqiao, Sheshan, and Maogang. Exclusion criteria were as follows: unable or unwilling to provide a written informed consent form; pregnancy; previously diagnosed critical illness, including cancer, stroke, CAD, cirrhosis, chronic hepatitis, cardiorespiratory failure, and hyper-or hypothyroidism; or have got organ transplantation or on dialysis therapy. For the present analysis, a total of 37,670 adults aged 20 to 74 years who were natives of Shanghai municipality or those have lived in Shanghai for at least 5 years were enrolled in the present study. Among these, we excluded participants who violated the inclusion criteria $(n=4271)$, who had no serum creatinine (Scr) measurement $(n=264)$ or had missing data on physical examination, questionnaire survey, or laboratory measurements $(n=1839)$. The final analysis included 31,296 participants (Fig. 1). The study protocol was approved by the Ethics Committee of Fudan University, School of Public Health (IRB\#2016-040586) and complied with the principles of the Declaration of Helsinki. Informed written consent were obtained from all participants prior to data collection.

\section{Data collection}

The general information of all study participants including sociodemographic characteristics (age, sex, marital status, educational level, and working status), selfreported history of chronic diseases (such as T2DM, hypertension, and cancer), and lifestyle factors (smoking status, alcohol consumption, and physical activity) was collected by trained interviewers through face-to-face interviews using a structured questionnaire. History of a diagnosis of T2DM, hypertension, cancer, stroke, CAD, 


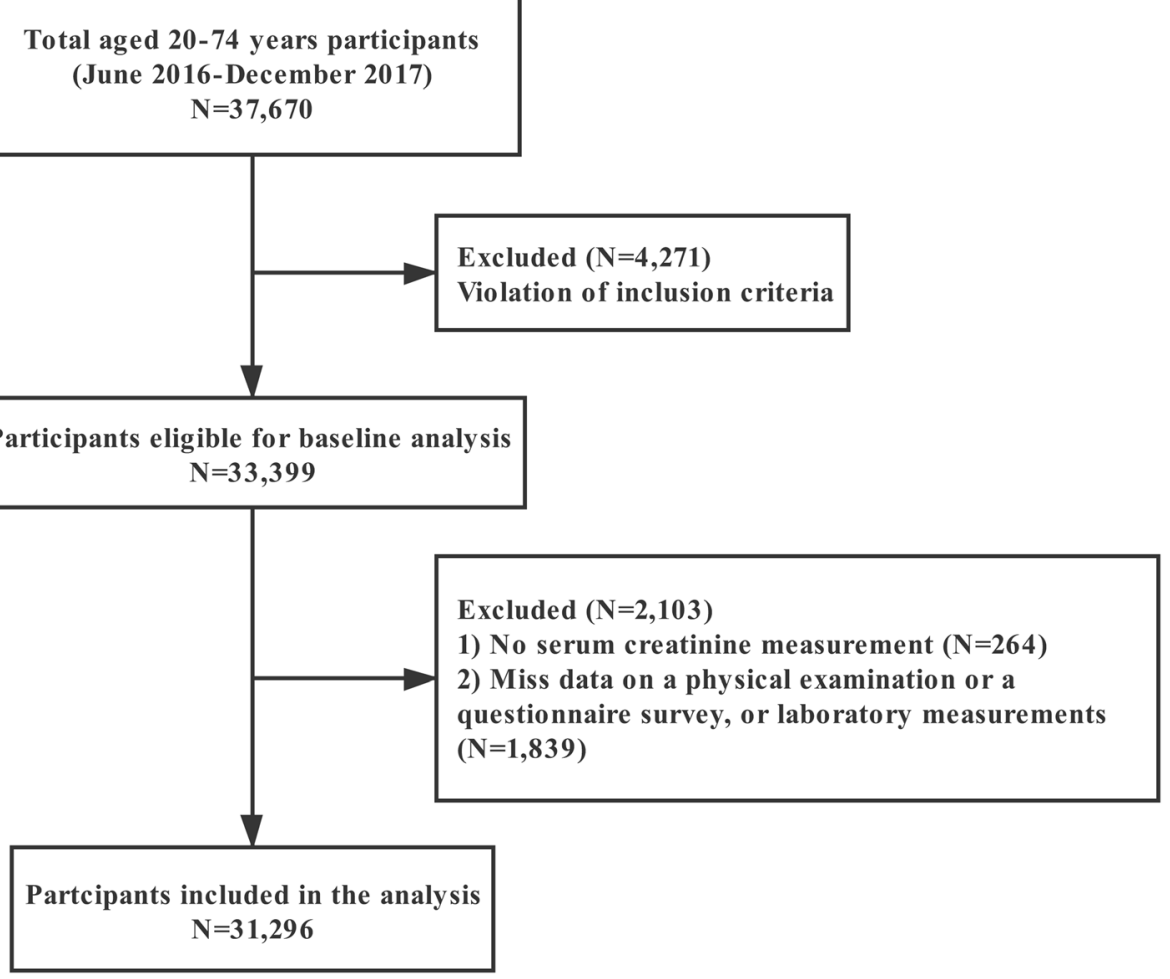

Fig. 1 Flowchart of the study population

cirrhosis, chronic hepatitis, cardiorespiratory failure, and hyper-or hypothyroidism, as well as receipt of organ transplantation or dialysis was obtained from electronic medical records. We used the International Physical Activity Questionnaire to assess physical activity. Smoking status was defined as $>1$ cigarette per day and lasting > 6 months, and alcohol consumption was defined as alcohol intake at least 3 times per week and lasting for at least half a year. Smoking status and alcohol consumption have been classified as never, former, or current.

Anthropometric data were obtained from all participants, including height, weight, and waist circumference (WC), and were measured in duplicate when participants were wearing light clothing without shoes. The mean values of these measurements were calculated for further analysis. Body mass index (BMI) was defined as body weight in kilograms divided by height in meters squared $\left(\mathrm{kg} / \mathrm{m}^{2}\right)$. Blood pressure (BP) was consecutively measured three times using an electronic sphygmomanometer, and the mean values were used for analysis.

\section{Laboratory measurement}

Prior to the investigation, all participants were asked to fast overnight for at least $8 \mathrm{~h}$, and fasting venous blood specimens were collected to perform laboratory measurements in DiAn medical laboratory center. Serum total cholesterol, TG, high-density lipoprotein (HDL) cholesterol, and low- density lipoprotein (LDL) cholesterol levels were measured using an automatic biochemical analyzer (Roche Cobas C501). Fasting plasma glucose (FPG) level was measured by glycokinase method using Roche P800 biochemical analyzer. Scr level was measured using enzymatic methods by Roche C702 automatic biochemical analyzer. HbA1c level was determined using high pressure liquid chromatography (TOSOH G8 automatic biochemical analyzer).

\section{Kidney function assessment}

The estimated glomerular fltration rate (eGFR) was calculated using the Chronic Kidney Disease Epidemiology Collaboration (CKD-EPI) equation for Chinese population [23]:

$$
\begin{aligned}
\operatorname{eGFR}\left(\mathrm{mL} / \mathrm{min} / 1.73 \mathrm{~m}^{2}\right)= & 141 \times \min (\mathrm{Scr} / \kappa, 1)^{\alpha} \\
& \times \max (\mathrm{Scr} / \kappa, 1)^{-1.209} \\
& \times 0.993^{\text {Age }} \\
& \times 1.018[\text { if female }]
\end{aligned}
$$

where Scr is the serum creatinine $(\mathrm{mg} / \mathrm{dl}), k$ is $0.7 \mathrm{for}$ females and 0.9 for males, $\alpha$ is -0.329 for females and 0.411 for males, min indicates the minimum of Scr $/ k$ or 1 , and max indicates the maximum of Scr/ $k$ or 1 .

Decreased eGFR was defined as an eGFR value below $60 \mathrm{~mL} / \mathrm{min} / 1.73 \mathrm{~m}^{2}$. The CKD classification was in accordance with the National Kidney Foundation [2], and 
we classified GFR stages into 4 categories as follows: normal eGFR, $\geq 90 \mathrm{~mL} / \mathrm{min} / 1.73 \mathrm{~m}^{2}$; mildly decreased eGFR, $\quad 60-89 \mathrm{~mL} / \mathrm{min} / 1.73 \mathrm{~m}^{2}$; moderately decreased eGFR, $30-59 \mathrm{~mL} / \mathrm{min} / 1.73 \mathrm{~m}^{2}$; and severely decreased eGFR, $15-29 \mathrm{~mL} / \mathrm{min} / 1.73 \mathrm{~m}^{2}$. In addition, eGFR was evaluated using the Modification of Diet in Renal Disease (MDRD) Study Equation for Chinese population [24] in a sensitivity analysis:

$$
\begin{aligned}
\mathrm{eGFR}_{\mathrm{MDRD}}\left(\mathrm{ml} / \mathrm{min} / 1.73 \mathrm{~m}^{2}\right) \\
=175 \times \text { serum creatinine }(\mathrm{mg} / \mathrm{dl})^{-1.154} \\
\times \text { age }^{-0.203} \times 0.742[\text { if female }]
\end{aligned}
$$

\section{Definitions of triglyceride waist phenotype, T2DM, and hypertension}

Participants were classified into four groups according to the following cut-off points [13]: (1) NTNW, normal serum TG level $(<1.7 \mathrm{mmol} / \mathrm{L})$ and normal WC $(<90 \mathrm{~cm}$ for men and $<80 \mathrm{~cm}$ for women); (2) NTGW, normal serum TG level and enlarged WC $(\geq 90 \mathrm{~cm}$ for men and $\geq$ $80 \mathrm{~cm}$ for women); (3) HTNW, elevated serum TG level ( $\geq 1.7 \mathrm{mmol} / \mathrm{L}$ ) and normal WC; (4) HTGW, elevated serum TG level and enlarged WC. In the sensitivity analyses, HTGW phenotype was also defined as elevated serum TG level $(\geq 2.0 \mathrm{mmol} / \mathrm{L})$ along with enlarged WC ( $\geq 90 \mathrm{~cm}$ for men and $\geq 85 \mathrm{~cm}$ for women) [15], or a TG level $\geq 2.0 \mathrm{mmol} / \mathrm{L}$ and $\mathrm{WC} \geq 90 \mathrm{~cm}$ for men or a TG level $\geq 1.5 \mathrm{mmol} / \mathrm{L}$ and $\mathrm{WC} \geq 85 \mathrm{~cm}$ for women [25].

The definition of T2DM was in accordance with the American Diabetes Association criteria [26]: FPG level $\geq$ $7.0 \mathrm{mmol} / \mathrm{L}$, HbA1c concentration $\geq 6.5 \%$, or previously diagnosed type 2 diabetes mellitus (T2DM). The diagnosis of hypertension was according to the Seventh Joint National Committee Report on Detection, Evaluation, and Treatment of High Blood Pressure guidelines (JNC7) [27]: systolic $\mathrm{BP} \geq 140 \mathrm{mmHg}$, diastolic $\mathrm{BP} \geq 90 \mathrm{mmHg}$, or previously diagnosed hypertension.

\section{Statistical analysis}

We accounted for a complex sample survey design, and the results were weighted in the present study. Continuous variables were presented as means \pm standard deviation (SD) or median with interquartile ranges. Categorical variables were expressed as number and percentage. We compared the differences between decreased eGFR and nondecreased eGFR using student's t test or Mann-Whitney U test for comparisons of continuous data and $\chi^{2}$ test for categorical data. We used weighted logistic regression models to determine the association of triglyceride waist phenotypes with decreased eGFR, and odd ratios (ORs) and 95\% confidence intervals (CIs) were calculated, with NTNW as the reference group. Multiple models were adjusted for age, sex (men vs. women), marital status (married vs. unmar$\mathrm{ried} /$ divorced/widowed), educational level (0-6, 7-12, and > 12 years), working status (retired vs. not retired), smoking status (never, former, and current), alcohol consumption (never, former, and current), physical activity, BMI, hypertension, LDL cholesterol, HDL cholesterol, total cholesterol, and T2DM.

For sensitivity analyses, we repeated the analyses to examine the association of triglyceride waist phenotypes with decreased eGFR and estimated GFR by the use of the MDRD equation or redefined the triglyceride waist phenotypes on the basis of the previous recommended criteria. We performed stratified analyses and potential effect modifications by sex, age ( $<60$ years, $\geq 60$ years), BMI $(<$ $24 \mathrm{~kg} / \mathrm{m}^{2}, \geq 24 \mathrm{~kg} / \mathrm{m}^{2}$ ), and presence or absence of T2DM or hypertension. In addition, we investigated the associations of four phenotype groups with the severity of decreased eGFR, including mildly, moderately, and severely decreased eGFR with using weighted multinomial logistic regression models, respectively by treating the normal eGFR group as the control group, and the same confounding factors as above were adjusted for the analyses.

All analyses were performed using SAS 9.4 version (Institute Inc., Cary, NC, USA). $P$ values of less than 0.05 (two-sided) were considered to be statistically significance.

\section{Results}

Baseline characteristics of subjects with or without decreased eGFR

Baseline characteristics of the study participants based on decreased eGFR status were shown in Table 1. Among 31, 296 participants, the mean age of the study participants was $55.64 \pm 11.35$ years, $12,702(40.59 \%)$ of them were men, 4247 (13.57\%) had T2DM, 15,881 (50.74\%) had hypertension. As expected, participants with decreased eGFR were more likely to have HTGW phenotype than those without decreased eGFR. Subjects with decreased eGFR had higher Scr, BMI, WC, BP, total cholesterol, TG, LDL cholesterol, and FPG levels, and a lower eGFR level in comparison with those in non-decreased eGFR subjects. In addition, less education, a higher proportion in past smokers and past drinkers were observed in decreased eGFR group than in non-decreased eGFR group. No significant differences in HDL cholesterol, marital status and physical activity were observed between the two groups.

\section{Association of triglyceride waist phenotypes with decreased eGFR}

Table 2 showed the ORs and 95\% CIs for the association of decreased eGFR with triglyceride waist phenotypes. Compared with the NTNW phenotype, NTGW and HTGW phenotypes were associated with a higher risk of decreased eGFR after adjusting for age, sex, marital status, educational level, working status, smoking status, 
Table 1 Characteristics of study participants by decreased eGFR

\begin{tabular}{|c|c|c|c|c|}
\hline Variables & Total & Decreased eGFR & Non-decreased eGFR & $\boldsymbol{P}$ value \\
\hline Men/Women & $12,702 / 18,594$ & $350 / 479$ & $12,352 / 18,115$ & 0.333 \\
\hline Age, years & $55.64 \pm 11.35$ & $64.10 \pm 7.23$ & $55.41 \pm 11.36$ & $<0.001$ \\
\hline Serum creatinine, mg/dl & $0.80 \pm 0.24$ & $1.40 \pm 0.96$ & $0.78 \pm 0.16$ & $<0.001$ \\
\hline $\mathrm{eGFR}, \mathrm{ml} / \mathrm{min} / 1.73 \mathrm{~m}^{2}$ & $92.75 \pm 15.33$ & $50.95 \pm 10.13$ & $93.88 \pm 13.77$ & $<0.001$ \\
\hline Body mass index, $\mathrm{kg} / \mathrm{m}^{2}$ & $24.35 \pm 3.34$ & $25.40 \pm 3.25$ & $24.32 \pm 3.34$ & $<0.001$ \\
\hline Waist circumference, cm & $81.50 \pm 9.40$ & $86.43 \pm 9.24$ & $81.36 \pm 9.37$ & $<0.001$ \\
\hline Systolic blood pressure, mmHg & $133.29 \pm 19.37$ & $144.40 \pm 20.91$ & $133.00 \pm 19.23$ & $<0.001$ \\
\hline Diastolic blood pressure, $\mathrm{mmHg}$ & $80.05 \pm 10.52$ & $81.95 \pm 10.85$ & $80.00 \pm 10.51$ & $<0.001$ \\
\hline Total cholesterol, mmol/L & $4.94 \pm 0.93$ & $5.19 \pm 1.00$ & $4.94 \pm 0.93$ & $<0.001$ \\
\hline Triglycerides, mmol/L & $1.35(0.98-1.92)$ & $1.53(1.12-2.17)$ & $1.34(0.97-1.91)$ & $<0.001$ \\
\hline $\mathrm{HDL}$ cholesterol, mmol/L & $1.41 \pm 0.36$ & $1.39 \pm 0.40$ & $1.41 \pm 0.35$ & 0.106 \\
\hline LDL cholesterol, mmol/L & $2.78 \pm 0.83$ & $2.97 \pm 0.92$ & $2.78 \pm 0.83$ & $<0.001$ \\
\hline Fasting glucose, mmol/L & $4.72(4.26-5.36)$ & $4.82(4.35-5.52)$ & $4.72(4.25-5.36)$ & $<0.001$ \\
\hline Marital status, n (\%) & & & & 0.597 \\
\hline Married & $29,137(93.10)$ & $768(92.64)$ & $28,369(93.11)$ & \\
\hline Unmarried/Divorced/Widowed & $2159(6.90)$ & $61(7.36)$ & $2098(6.89)$ & \\
\hline Educational level, years & & & & $<0.001$ \\
\hline$\leq 6^{a}$ & $14,068(44.95)$ & $538(64.90)$ & $13,530(44.41)$ & \\
\hline $7-12^{\mathrm{a}}$ & $15,028(48.02)$ & $280(33.78)$ & $14,748(48.41)$ & \\
\hline$\geq 13^{\mathrm{a}}$ & $2200(7.03)$ & $11(1.33)$ & $2189(7.18)$ & \\
\hline Working status, n (\%) & & & & $<0.001$ \\
\hline Not retired $^{\mathrm{a}}$ & $13,667(43.67)$ & $149(17.97)$ & $13,518(44.37)$ & \\
\hline Retired $^{a}$ & $17,629(56.33)$ & $680(82.03)$ & $16,949(55.63)$ & \\
\hline Smoking status, n (\%) & & & & $<0.001$ \\
\hline Never & 23,917 (76.42) & $645(77.80)$ & $23,272(76.38)$ & \\
\hline Former $^{a}$ & 1048 (3.35) & $51(6.15)$ & 997 (3.27) & \\
\hline Current $^{a}$ & $6331(20.23)$ & $133(16.04)$ & $6198(20.34)$ & \\
\hline Alcohol consumption, n (\%) & & & & 0.002 \\
\hline Never & $27,064(86.48)$ & 724 (87.33) & $26,340(86.45)$ & \\
\hline Former $^{a}$ & $282(0.90)$ & $16(1.93)$ & $266(0.87)$ & \\
\hline Current & $3950(12.62)$ & 89 (10.74) & $3861(12.67)$ & \\
\hline Physical activity, MET-min/week & $2351.42 \pm 591.27$ & $2372.90 \pm 593.80$ & $2350.80 \pm 591.20$ & 0.290 \\
\hline Type 2 diabetes mellitus, n (\%) & $4247(13.57)$ & $167(20.14)$ & $4080(13.39)$ & $<0.001$ \\
\hline Hypertension, n (\%) & $15,881(50.74)$ & $634(76.48)$ & $15,247(50.04)$ & $<0.001$ \\
\hline Hypertriglyceridemic waist phenotype, n (\%) & & & & $<0.001$ \\
\hline NTNW $^{a}$ & $13,905(44.43)$ & $213(25.69)$ & $13,692(44.94)$ & \\
\hline NTGW & $7179(23.00)$ & $273(32.93)$ & $6924(22.73)$ & \\
\hline HTNW & $4806(15.36)$ & 109 (13.15) & $4697(15.42)$ & \\
\hline HTGW ${ }^{a}$ & $5388(17.22)$ & $234(28.23)$ & $5154(16.92)$ & \\
\hline
\end{tabular}

Data are presented as mean $\pm S D$, median (interquartile range), or number (\%)

eGFR Estimated glomerular filtration rate, HDL High-density lipoprotein, LDL Low-density lipoprotein, MET Metabolic equivalent task, NWNT Normal triglyceride level $(<1.7 \mathrm{mmol} / \mathrm{L})$ and normal waist circumference $(<90 \mathrm{~cm}$ for men and $<80 \mathrm{~cm}$ for women), NTGW Normal triglyceride level and enlarged waist circumference $(\geq 90 \mathrm{~cm}$ for men and $\geq 80 \mathrm{~cm}$ for women), HTNW Elevated triglyceride level $(\geq 1.7 \mathrm{mmol} / \mathrm{L})$ and normal waist circumference, HTGW Elevated triglyceride level and enlarged waist circumference

${ }^{\mathrm{a}} p<0.05$ between decreased eGFR and non-decreased eGFR 
Table 2 Odds ratios for decreased eGFR according to triglyceride level and waist circumference

\begin{tabular}{|c|c|c|c|c|c|c|c|}
\hline \multirow[t]{2}{*}{ Variables } & \multirow{2}{*}{$\begin{array}{l}\text { Cases/ } \\
\text { Participants }\end{array}$} & Model $1^{a}$ & \multirow[t]{2}{*}{$P$ value } & Model $2^{\mathbf{b}}$ & \multirow[t]{2}{*}{$P$ value } & Model $3^{c}$ & \multirow[t]{2}{*}{$P$ value } \\
\hline & & OR (95\% Cl) & & OR $(95 \% \mathrm{Cl})$ & & OR $(95 \% \mathrm{Cl})$ & \\
\hline \multicolumn{8}{|c|}{ Decreased eGFR } \\
\hline NTNW & 213/13,905 & 1.00 & & 1.00 & & 1.00 & \\
\hline NTGW & 273/7197 & $1.94(1.60-2.35)$ & $<0.001$ & $1.94(1.60-2.34)$ & $<0.001$ & $1.75(1.41-2.18)$ & $<0.001$ \\
\hline HTNW & $109 / 4806$ & $1.55(1.22-1.96)$ & $<0.001$ & $1.55(1.23-1.96)$ & $<0.001$ & $1.29(0.99-1.68)$ & 0.060 \\
\hline HTGW & $234 / 5388$ & $2.54(2.09-3.09)$ & $<0.001$ & $2.55(2.09-3.10)$ & $<0.001$ & $1.99(1.54-2.58)$ & $<0.001$ \\
\hline$P$-trend & & $<0.001$ & & $<0.001$ & & $<0.001$ & \\
\hline \multicolumn{8}{|c|}{ Decreased eGFR } \\
\hline NTNW & $351 / 13,905$ & 1.00 & & 1.00 & & 1.00 & \\
\hline NTGW & $402 / 7197$ & $1.81(1.56-2.11)$ & $<0.001$ & $1.82(1.56-2.12)$ & $<0.001$ & $1.79(1.50-2.13)$ & $<0.001$ \\
\hline HTNW & $150 / 4806$ & $1.24(1.02-1.51)$ & 0.029 & $1.24(1.02-1.51)$ & 0.029 & $1.11(0.89-1.39)$ & 0.348 \\
\hline HTGW & $316 / 5388$ & $2.07(1.77-2.43)$ & $<0.001$ & $2.09(1.78-2.45)$ & $<0.001$ & $1.88(1.52-2.34)$ & $<0.001$ \\
\hline$P$-trend & & $<0.001$ & & $<0.001$ & & $<0.001$ & \\
\hline
\end{tabular}

eGFR Estimated glomerular filtration rate, OR Odds ratio, Cl Confidence interval, MDRD Modification of Diet in Renal Disease, NWNT Normal triglyceride level $(<1.7$ $\mathrm{mmol} / \mathrm{L}$ ) and normal waist circumference $(<90 \mathrm{~cm}$ for men and $<80 \mathrm{~cm}$ for women), NTGW Normal triglyceride level and enlarged waist circumference $(\geq 90 \mathrm{~cm}$ for men and $\geq 80 \mathrm{~cm}$ for women), HTNW Elevated triglyceride level $(\geq 1.7 \mathrm{mmol} / \mathrm{L})$ and normal waist circumference, HTGW Elevated triglyceride level and enlarged waist circumference

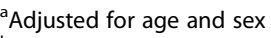

${ }^{\mathrm{b}}$ Adjusted for model 1 covariates plus marital status, educational level, working status, smoking status, alcohol consumption, and physical activity

'Adjusted for model 2 covariates plus body mass index, hypertension, low-density lipoprotein (LDL) cholesterol, high-density lipoprotein (HDL) cholesterol, total cholesterol, and T2DM

alcohol consumption, physical activity, BMI, hypertension, LDL cholesterol, HDL cholesterol, total cholesterol, and T2DM; whereas HTNW phenotype was not significantly associated with decreased eGFR risk. The multivariable-adjusted ORs for decreased eGFR risk associated with NTGW, HTNW, and HTGW phenotypes were 1.75 (95\% CI, 1.41-2.18 $P<0.001$ ), 1.29 (95\% CI, $0.99-1.68, P=0.060$ ), and 1.99 (95\% CI, 1.54-2.58, $\mathrm{P}<0.001$ ), respectively. We also performed three sensitivity analyses including the MDRD equation-based eGFR and redefining the triglyceride waist phenotypes to assess the robustness of our results, and found similar results in all sensitivity analyses (Tables 2 and 3 ).

We further examined the association of triglyceride waist phenotypes with decreased eGFR in different subgroups of sex, age, BMI, T2DM, and hypertension in Table 4. The associations between NTGW, HTNW, and HTGW phenotypes and the risk of decreased eGFR remained consistent across almost all subgroups. The strongest positive association of HTGW phenotype with decreased eGFR was found in the subgroup of presence of T2DM (OR 2.60, 95\% CI 1.38-4.89). No significant interaction effect was observed between the triglyceride waist phenotypes and all subgroup variables in decreased eGFR risk.

\section{Association of different triglyceride waist phenotypes with mildly, moderately, and severely decreased eGFR} The multivariable-adjusted ORs for mildly, moderately, and severely decreased eGFR according to triglyceride waist phenotypes were present in Table 5 . The number of participants with normal eGFR, mildly decreased eGFR, moderately decreased eGFR, and severely decreased eGFR were 19,538, 10,929, 785, and 30, respectively. After adjusting for potential confounders, HTGW phenotype was positively associated with mildly decreased eGFR risk, whereas no significant association of NTGW and HTNW phenotypes with mildly decreased eGFR was found. Risk of moderately decreased eGFR was higher for subjects with NTGW and HTGW phenotypes, but not for subjects with HTNW phenotype, as compared to subjects with NTNW phenotype. This was consistent with the results of primary analyses among all subjects. No significant association was found between NTGW, HTNW, and HTGW phenotypes and the risk of severely decreased eGFR.

\section{Discussion}

In this large population-based, cross-sectional study, we explored the association of triglyceride waist phenotypes with decreased eGFR $\left(<60 \mathrm{~mL} / \mathrm{min} / 1.73 \mathrm{~m}^{2}\right)$ in the overall population and across a variety of subgroups. We found that HTGW phenotype was associated an increased risk of decreased eGFR; in addition, HTGW phenotype was significantly associated with the progression of renal function decline except for severely decreased eGFR. To the best of our knowledge, this is the first study to examine the association of HTGW phenotype with different stages of renal function in Chinese adults. 
Table 3 Odds ratios for decreased eGFR at different levels of triglyceride level and waist circumference

\begin{tabular}{|c|c|c|c|c|c|c|c|}
\hline \multirow[t]{2}{*}{ Variables } & \multirow{2}{*}{$\begin{array}{l}\text { Cases/ } \\
\text { Participants }\end{array}$} & Model $1^{\mathbf{a}}$ & \multirow[t]{2}{*}{$P$ value } & Model $2^{\mathbf{b}}$ & \multirow[t]{2}{*}{$P$ value } & Model $3^{c}$ & \multirow[t]{2}{*}{$P$ value } \\
\hline & & OR (95\% Cl) & & OR (95\% Cl) & & OR (95\% Cl) & \\
\hline \multicolumn{8}{|c|}{ Definition 1} \\
\hline NTNW1 & $317 / 18,318$ & 1.00 & & 1.00 & & 1.00 & \\
\hline NTGW1 & $250 / 5808$ & $1.80(1.52-2.14)$ & $<0.001$ & $1.80(1.51-2.14)$ & $<0.001$ & $1.69(1.38-2.07)$ & $<0.001$ \\
\hline HTNW1 & $107 / 4228$ & $1.52(1.22-1.90)$ & $<0.001$ & $1.52(1.21-1.90)$ & $<0.001$ & $1.35(1.04-1.76)$ & 0.026 \\
\hline HTGW1 & $155 / 2942$ & $2.78(2.28-3.40)$ & $<0.001$ & $2.80(2.29-3.41)$ & $<0.001$ & $2.42(1.84-3.17)$ & $<0.001$ \\
\hline$P$-trend & & $<0.001$ & & $<0.001$ & & $<0.001$ & \\
\hline \multicolumn{8}{|c|}{ Definition 2} \\
\hline NTNW2 & 269/16,019 & 1.00 & & 1.00 & & 1.00 & \\
\hline NTGW2 & $196 / 4548$ & $1.89(1.56-2.28)$ & $<0.001$ & $1.88(1.55-2.27)$ & $<0.001$ & $1.76(1.41-2.19)$ & $<0.001$ \\
\hline HTNW2 & $155 / 6527$ & $1.50(1.22-1.84)$ & $<0.001$ & $1.49(2.21-1.83)$ & $<0.001$ & $1.26(1.00-1.60)$ & 0.054 \\
\hline HTGW2 & 209/4202 & $2.57(2.12-3.11)$ & $<0.001$ & $2.58(2.13-3.12)$ & $<0.001$ & $2.10(1.62-2.71)$ & $<0.001$ \\
\hline$P$-trend & & $<0.001$ & & $<0.001$ & & $<0.001$ & \\
\hline
\end{tabular}

eGFR Estimated glomerular filtration rate, OR Odds ratio, CI Confidence interval, NWNT1 Normal triglyceride level $(<2.0 \mathrm{mmol} / \mathrm{L})$ and normal waist circumference $(<90 \mathrm{~cm}$ for men and $<85 \mathrm{~cm}$ for women), NTGW1 Normal triglyceride level and enlarged waist circumference $(\geq 90 \mathrm{~cm}$ for men and $\geq 85 \mathrm{~cm}$ for women), HTNW1 Elevated triglyceride level $(\geq 2.0 \mathrm{mmol} / \mathrm{L})$ and normal waist circumference, HTGW1 Elevated triglyceride level and enlarged waist circumference, NWNT2 Normal triglyceride level $(<2.0 \mathrm{mmol} / \mathrm{L}$ for men and $<1.5 \mathrm{mmol} / \mathrm{L}$ for women) and normal waist circumference $(<90 \mathrm{~cm}$ for men and $<85 \mathrm{~cm}$ for women), NTGW2 Normal triglyceride level and enlarged waist circumference $(\geq 90 \mathrm{~cm}$ for men and $\geq 85 \mathrm{~cm}$ for women), HTNW2 Elevated triglyceride level $(\geq 2.0 \mathrm{mmol} / \mathrm{L}$ for men and $\geq 1.5$ $\mathrm{mmol} / \mathrm{L}$ for women) and normal waist circumference, HTGW2 Elevated triglyceride level and enlarged waist circumference

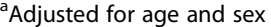

${ }^{\mathrm{b}}$ Adjusted for model 1 covariates plus marital status, educational level, working status, smoking status, alcohol consumption, and physical activity 'Adjusted for model 2 covariates plus body mass index, hypertension, low-density lipoprotein (LDL) cholesterol, high-density lipoprotein (HDL) cholesterol, total cholesterol, and T2DM

Table 4 Odds ratios for decreased eGFR according to triglyceride level and waist circumference by various subpopulations

\begin{tabular}{|c|c|c|c|c|c|c|c|}
\hline Subpopulation & Cases/Participants & NTNW & NTGW & HTNW & HTGW & P-trend & P-interaction \\
\hline Sex groups & & & & & & & 0.637 \\
\hline Men & $350 / 12,702$ & 1.00 & $1.66(1.19-2.33)$ & $1.30(0.92-1.85)$ & $1.76(1.17-2.64)$ & 0.016 & \\
\hline Women & $479 / 18,594$ & 1.00 & $1.83(1.36-2.45)$ & $1.21(0.80-1.85)$ & $2.27(1.61-3.20)$ & $<0.001$ & \\
\hline Age, years & & & & & & & 0.642 \\
\hline$<60$ & $197 / 18,111$ & 1.00 & $2.00(1.28-3.12)$ & $1.33(0.82-2.17)$ & $1.96(1.17-3.26)$ & 0.052 & \\
\hline$\geq 60$ & $632 / 13,185$ & 1.00 & $1.74(1.35-2.23)$ & $1.29(0.94-1.78)$ & $2.09(1.54-2.83)$ & $<0.001$ & \\
\hline $\mathrm{BMI}, \mathrm{kg} / \mathrm{m}^{2}$ & & & & & & & 0.431 \\
\hline$<24$ & $288 / 14,965$ & 1.00 & $2.09(1.48-2.95)$ & $1.29(0.86-1.93)$ & $1.84(1.10-3.08)$ & 0.008 & \\
\hline$\geq 24$ & $541 / 16,331$ & 1.00 & $1.73(1.28-2.34)$ & $1.37(0.93-2.02)$ & $2.06(1.47-2.89)$ & $<0.001$ & \\
\hline Presence of T2DM & & & & & & & 0.412 \\
\hline No & $662 / 27,049$ & 1.00 & $1.63(1.28-2.07)$ & $1.29(0.96-1.73)$ & $2.00(1.50-2.67)$ & $<0.001$ & \\
\hline Yes & $167 / 4247$ & 1.00 & $2.90(1.65-5.12)$ & $1.49(0.76-2.89)$ & $2.60(1.38-4.89)$ & 0.098 & \\
\hline Presence of hypertension & & & & & & & 0.542 \\
\hline No & $195 / 15,415$ & 1.00 & $1.58(1.04-2.39)$ & $0.92(0.52-1.60)$ & $1.70(0.99-2.92)$ & 0.155 & \\
\hline Yes & $634 / 15,881$ & 1.00 & $1.84(1.42-2.38)$ & $1.46(1.07-1.98)$ & $2.14(1.58-2.88)$ & $<0.001$ & \\
\hline
\end{tabular}

All models adjusted for age, sex, marital status, educational level, working status, smoking status, alcohol consumption, physical activity, body mass index, hypertension, low-density lipoprotein (LDL) cholesterol, high-density lipoprotein (HDL) cholesterol, total cholesterol, and T2DM

eGFR estimated glomerular filtration rate, BMI Body mass index, T2DM Type 2 diabetes mellitus, NWNT Normal triglyceride level $(<1.7 \mathrm{mmol} / \mathrm{L})$ and normal waist circumference $(<90 \mathrm{~cm}$ for men and $<80 \mathrm{~cm}$ for women), NTGW Normal triglyceride level and enlarged waist circumference $(\geq 90 \mathrm{~cm}$ for men and $\geq 80 \mathrm{~cm}$ for women), HTNW Elevated triglyceride level ( $\geq 1.7 \mathrm{mmol} / \mathrm{L})$ and normal waist circumference, $H T G W$ Elevated triglyceride level and enlarged waist circumference 
Table 5 Odds ratios for mildly, moderately, and severely decreased eGFR according to triglyceride level and waist circumference

\begin{tabular}{|c|c|c|c|c|c|c|c|c|}
\hline & NTNW & NTGW & $\boldsymbol{P}$ value & HTNW & $\boldsymbol{P}$ value & HTGW & $\boldsymbol{P}$ value & P-trend \\
\hline \multicolumn{9}{|l|}{ Mildly decreased eGFR } \\
\hline Number of participants & 4368 & 2784 & & 1684 & & 2093 & & \\
\hline Model $1^{\mathrm{a}}$ & 1.00 & $1.12(1.05-1.20)$ & $<0.001$ & $1.16(1.08-1.25)$ & $<0.001$ & $1.26(1.18-1.36)$ & $<0.001$ & $<0.001$ \\
\hline Model $2^{\mathrm{b}}$ & 1.00 & $1.13(1.06-1.21)$ & $<0.001$ & $1.16(1.08-1.26)$ & $<0.001$ & $1.28(1.19-1.37)$ & $<0.001$ & $<0.001$ \\
\hline Model $3^{c}$ & 1.00 & $1.05(0.97-1.13)$ & 0.262 & $1.07(0.98-1.17)$ & 0.145 & $1.13(1.02-1.24)$ & 0.020 & 0.020 \\
\hline \multicolumn{9}{|c|}{ Moderately decreased eGFR } \\
\hline Number of participants & 200 & 260 & & 99 & & 226 & & \\
\hline Model $1^{\mathrm{a}}$ & 1.00 & $2.07(1.70-2.53)$ & $<0.001$ & $1.60(1.25-2.05)$ & $<0.001$ & $2.90(2.37-3.56)$ & $<0.001$ & $<0.001$ \\
\hline Model $2^{\mathrm{b}}$ & 1.00 & $2.08(1.70-2.54)$ & $<0.001$ & $1.60(1.25-2.05)$ & $<0.001$ & $2.93(2.39-3.60)$ & $<0.001$ & $<0.001$ \\
\hline Model $3^{c}$ & 1.00 & $1.81(1.44-2.27)$ & $<0.001$ & $1.32(1.00-1.75)$ & 0.051 & $2.23(1.70-2.92)$ & $<0.001$ & $<0.001$ \\
\hline \multicolumn{9}{|l|}{ Severely decreased eGFR } \\
\hline Number of participants & 7 & 10 & & 6 & & 7 & & \\
\hline Model $1^{\mathrm{a}}$ & 1.00 & $2.01(0.74-5.48)$ & 0.173 & $2.79(0.93-8.34)$ & 0.066 & $2.31(0.79-6.78)$ & 0.129 & 0.084 \\
\hline Model $2^{b}$ & 1.00 & $2.06(0.75-5.64)$ & 0.159 & $2.86(0.95-8.55)$ & 0.061 & $2.35(0.80-6.90)$ & 0.121 & 0.078 \\
\hline Model $3^{c}$ & 1.00 & $1.50(0.48-4.71)$ & 0.487 & $1.57(0.45-5.49)$ & 0.481 & $1.10(0.28-4.33)$ & 0.890 & 0.938 \\
\hline
\end{tabular}

eGFR Estimated glomerular filtration rate, NWNT Normal triglyceride level $(<1.7 \mathrm{mmol} / \mathrm{L})$ and normal waist circumference $(<90 \mathrm{~cm}$ for men and $<80 \mathrm{~cm}$ for women), NTGW Normal triglyceride level and enlarged waist circumference ( $\geq 90 \mathrm{~cm}$ for men and $\geq 80 \mathrm{~cm}$ for women), HTNW Elevated triglyceride level $(\geq 1.7$ $\mathrm{mmol} / \mathrm{L}$ ) and normal waist circumference, HTGW Elevated triglyceride level and enlarged waist circumference

${ }^{a}$ Adjusted for age and sex

${ }^{\mathrm{b}}$ Adjusted for model 1 covariates plus marital status, educational level, working status, smoking status, alcohol consumption, and physical activity

'Adjusted for model 2 covariates plus body mass index, hypertension, low-density lipoprotein (LDL) cholesterol, high-density lipoprotein (HDL) cholesterol, total cholesterol, and T2DM

HTGW phenotype was found to have a similar predictive power with metabolic syndrome (MetS), and may be more easily applicable than MetS [28]. A recent cross-sectional study has shown that the HTGW phenotype, and not the common indices (including WC, waistto-hip ratio, and waist-to-height ratio), was associated with a higher risk of CKD [18]. The relationship between triglyceride waist phenotypes and CKD has been extensively reported in many cross-sectional studies. However, most studies were conducted in with a limited sample size [16-20] and findings were inconsistent. In a middleaged or older population, compared with NTNW phenotype, NTGW/HTNW and HTGW phenotypes were associated with higher risk of CKD [16]. Results regarding association of triglyceride waist phenotypes with CKD in the cross-sectional study and prospective study were not identical [19]. The reasons for the conflicting results may likely be due to the difference of population characteristics, the number of participants, inclusion of confounding factors, and methods of eGFR calculation. Ramezankhani et al. [19] and our study estimated GFR using the CKD-EPI equation, which was a more accurate method of renal function than the MDRD Equation in routine clinical practice. While many previous studies [16-18, 20] used the MDRD Equation for estimating GFR, a most common method for estimating GFR.

The decline in eGFR was related with the increased risk of mortality and end-stage renal disease (ESRD)
[29]. Only a few studies have determined the association of decreased eGFR risk with triglyceride waist phenotypes [21], and shown that HTGW was associated with abnormal renal function among both Chinese and Australian subjects. In the present large Chinese population, we also observed similar findings in overall and stratified populations; NTGW phenotype was also associated with an increased risk of reduced eGFR. In the stratified analyses, the independent positive association of NTGW and HTGW phenotypes with reduced eGFR risk still persisted across almost all subgroups; a strongest association between HTGW phenotype and decreased eGFR risk was found in the subgroup of presence of T2DM, suggesting the predictive power of HTGW for decreased eGFR might be better for T2DM patients. Our findings suggest that each of the triglyceride waist phenotypes may play different roles on renal function decline. This lack of evidence may be explained by differences of NTGW and HTNW phenotype on decreased eGFR, and it is in line with a previous study reporting associations with CKD in men [17]. TG was considered to be a useful marker of visceral obesity with a given WC [30]. Additionally, compared with NTGW or HTNW phenotype, HTGW phenotype was a more stable and stronger risk factor for renal function decline. Thus, our data provide evidence that HTGW phenotype might play an important role in the development of renal function impairment. 
Corresponding studies on the role of triglyceride waist phenotypes on the development of kidney dysfunction remain scarce. Most studies were performed to examine the association of HTGW phenotype with CKD risk, but were not involved in CKD progression. In the present study, we found that NTGW and HTGW phenotypes were independently associated with mildly decreased eGFR, or moderately decreased eGFR risk, except for the NTGW-mildly decreased eGFR association. No significant association between any of the phenotypes and severely decreased eGFR was found. Underlying mechanisms of these associations are still unknown, the null association of triglyceride waist phenotypes with severely decreased eGFR may be due to the small number of subjects. Thus, our findings suggest that HTGW phenotype may be an independent risk factor for the development of renal function impairment, prevention and that control of HTGW may be an effective measure to attenuate the risk of the progression of renal function decline. HTGW was closely related to visceral obesity, which may result in fat accumulation in kidney [30,31]. Excess accumulation of fat on the kidney, which is related to oxidative stress and inflammation response, may impair the kidney and contribute to an unfavorable renal hemodynamic profile [32]. CKD patients have a lower activity of lipoprotein lipase and hepatic TG lipase, which likely leads to the development of hypertriglyceridemia, and subsequently to augment renal impairment $[33,34]$. Further elucidation of the mechanism for the role of HTGW on renal function or CKD is still warranted.

The strengths of the present study include a largescale population-based study, enrollment of participants living in both urban and rural areas, and the extensive adjustment for potential confounders, including demographic, lifestyle, anthropometric, and clinical factors. Several potential limitations of our study also should be considered. Firstly, the causal relationship could not be determined due to the nature of cross-sectional study design in our analyses. Secondly, all study participants were from only one district of Shanghai, China, and the generalizability of our results may be limited. Further studies should be conducted in more diverse regions. Thirdly, although many covariates were adjusted for in our analyses, the present study still lacks data on medication treatment, dietary, and genetic factors, and so on. Lastly, despite the overall relatively large sample size, only a few participants were in the subpopulations, especially for severely decreased eGFR, which resulted in wide CIs for the effect estimates and inaccurate results.

\section{Conclusions}

In conclusion, HTGW phenotype was significantly associated with an increased risk of decreased eGFR in the overall study population, and remained consistent across all subgroups, which suggests that the HTGW phenotype may be a stable risk factor for the decline of kidney function. We also found that the HTGW phenotype was significantly associated with higher risks of mildly and moderately decreased eGFR, but not with severely decreased eGFR. These findings underscore the importance of preventing and controlling the HTGW phenotype, which may reduce the risk of kidney function decline or even the progression of CKD.

\section{Abbreviations}

BMI: Body mass index; BP: Blood pressure; CAD: Coronary artery disease; Cls: Confidence intervals; CKD: Chronic kidney disease; CKD-EPI: Chronic Kidney Disease Epidemiology Collaboration; CVD: Cardiovascular diseases; eGFR: Estimated glomerular filtration rate; ESRD: End-stage renal disease; FPG: Fasting plasma glucose; HDL: High-density lipoprotein; HTGW: Hypertriglyceridemic waist; HTNW: Elevated TG level/normal WC; LDL: Low-density lipoprotein; MDRD: Modification of Diet in Renal Disease; MetS: Metabolic syndrome; NTGW: Normal TG level/enlarged WC; NTNW: Normal TG level/normal WC; ORs: Odd ratios; Scr: Serum creatinine; TG: Triglycerides; T2DM: Type 2 diabetes mellitus; WC: Waist circumference

\section{Acknowledgments}

The authors thank all the study participants in this study, the leader of the Disease Control and Prevention Center in Songjiang District, Shanghai for assistance with organization and substantial support, and all the staffs of the Community Health Centers from Zhongshan, Xinqiao, Sheshan and Maogang in Songjiang District for their technical support and contribution of data collection.

\section{Authors' contributions}

$Y Q, Q Z$, and NW conceived and designed the study. YY, RW, YZ, and SC contributed to the data analysis and interpretation. $M Z, X L$, and $Y Q$ contributed to data acquisition and the manuscript draft. YJ and GZ supervised the study and revised the manuscript. All authors approved the final version of the manuscript to be submitted.

\section{Funding}

This work was supported by the National Key Research and Development Program of China, Precision Medicine Project (2017YFC0907000) and the Shanghai Municipal Education Commission-Gaofeng Discipline Development Project for Public Health and Preventive Medicine (No. 17).

\section{Availability of data and materials}

The dataset used and analyzed during the current study is available from the corresponding author on reasonable request.

\section{Ethics approval and consent to participate}

The study protocol was approved by the Ethics Committee of the Fudan University, School of Public Health (IRB\#2016-04-0586) and complied with the principles of the Declaration of Helsinki. Informed written consents were obtained from all participants before data collection.

\section{Consent for publication}

Not Applicable.

Competing interests

The authors have no conflicts of interest to declare.

Received: 11 April 2020 Accepted: 27 July 2020

Published online: 05 August 2020

\section{References}

1. Bikbov B, Purcell CA, Levey AS, Smith M, Abdoli A, Abebe M, et al. Global, regional, and national burden of chronic kidney disease, 1990-2017: a systematic analysis for the global burden of disease study 2017. Lancet. 2020;395(10225):709-33.

2. Levey AS, Coresh J, Balk E, Kausz AT, Levin A, Steffes MW, et al. National Kidney Foundation practice guidelines for chronic kidney disease: evaluation, classification, and stratification. Ann Intern Med. 2003;139(2):137-47 
3. Ene-lordache B, Perico N, Bikbov B, Carminati S, Remuzzi A, Perna A, et al. Chronic kidney disease and cardiovascular risk in six regions of the world (ISN-KDDC): a cross-sectional study. Lancet Glob Health. 2016;4(5):e307-19.

4. Z Zhang L, Wang F, Wang L, Wang W, Liu B, Liu J, et al. Prevalence of chronic kidney disease in China: a cross-sectional survey. Lancet. 2012;379(9818):815-22.

5. Nugent RA, Fathima SF, Feigl AB, Chyung D. The burden of chronic kidney disease on developing nations: a 21st century challenge in global health. Nephron Clin Pract. 2011;118(3):c269-77.

6. Hermans MM, Henry R, Dekker JM, Kooman JP, Kostense PJ, Nijpels G, et al. Estimated glomerular filtration rate and urinary albumin excretion are independently associated with greater arterial stiffness: the Hoorn study. J Am Soc Nephrol. 2007;18(6):1942-52.

7. Navaneethan SD, Schold JD, Arrigain S, Jolly SE, Nally JJ. Cause-specific deaths in non-Dialysis-dependent CKD. J Am Soc Nephrol. 2015;26(10): 2512-20.

8. Lemieux I, Pascot A, Couillard C, Lamarche B, Tchernof A, Almeras N, et al. Hypertriglyceridemic waist: a marker of the atherogenic metabolic triad (hyperinsulinemia; hyperapolipoprotein B; small, dense LDL) in men? Circulation. 2000;102(2):179-84.

9. Wang A, Li Z, Zhou Y, Wang C, Luo Y, Liu X, et al. Hypertriglyceridemic waist phenotype and risk of cardiovascular diseases in China: results from the Kailuan study. Int J Cardiol. 2014;174(1):106-9.

10. Blackburn P, Lemieux I, Lamarche B, Bergeron J, Perron P, Tremblay G, et al. Hypertriglyceridemic waist: a simple clinical phenotype associated with coronary artery disease in women. Metabolism. 2012;61(1):56-64.

11. Li Q, Zhang D, Guo C, Zhou Q, Tian G, Liu D, et al. Association of hypertriglyceridemic waist-to-height ratio and its dynamic status with incident hypertension: the rural Chinese cohort study. J Hypertens. 2019; 37(12):2354-60.

12. Zhao K, Yang SS, Wang HB, Chen K, Lu ZH, Mu YM. Association between the Hypertriglyceridemic waist phenotype and Prediabetes in Chinese adults aged 40 years and older. J Diabetes Res. 2018;2018:1031939.

13. Ren Y, Zhang M, Zhao J, Wang C, Luo X, Zhang J, et al. Association of the hypertriglyceridemic waist phenotype and type 2 diabetes mellitus among adults in China. J Diabetes Investig. 2016;7(5):689-94.

14. Chen S, Guo X, Dong S, Yu S, Chen Y, Zhansg N, et al. Association between the hypertriglyceridemic waist phenotype and hyperuricemia: a crosssectional study. Clin Rheumatol. 2017:36(5):1111-9.

15. Sam S, Haffner S, Davidson MH, D'Agostino RS, Feinstein S, Kondos G, et al. Hypertriglyceridemic waist phenotype predicts increased visceral fat in subjects with type 2 diabetes. Diabetes Care. 2009;32(10):1916-20.

16. Li Y, Zhou C, Shao X, Liu X, Guo J, Zhang Y, et al. Hypertriglyceridemic waist phenotype and chronic kidney disease in a Chinese population aged 40 years and older. PLoS One. 2014;9(3):e92322.

17. Zeng J, Liu M, Wu L, Wang J, Yang S, Wang Y, et al. The Association of Hypertriglyceridemic Waist Phenotype with chronic kidney disease and its sex difference: a cross-sectional study in an urban Chinese elderly population. Int J Environ Res Public Health. 2016;13(12):1233.

18. Zhou C, Li Y, Shao X, Zou H. Identification of chronic kidney disease risk in relatively lean southern Chinese: the hypertriglyceridemic waist phenotype vs. anthropometric indexes. Eat Weight Disord. 2018;23(6):885-92.

19. Ramezankhani A, Azizi F, Ghanbarian A, Parizadeh D, Hadaegh F. The hypertriglyceridemic waist and waist-to-height ratio phenotypes and chronic kidney disease: cross-sectional and prospective investigations. Obes Res Clin Pract. 2017;11:585-96.

20. Huang J, Zhou C, Li Y, Zhu S, Liu A, Shao X, et al. Visceral adiposity index, hypertriglyceridemic waist phenotype and chronic kidney disease in a southern Chinese population: a cross-sectional study. Int Urol Nephrol. 2015; 47(8):1387-96.

21. Yu D, Yang W, Chen T, Cai Y, Zhao Z, Simmons D. Hypertriglyceridemicwaist is more predictive of abnormal liver and renal function in an Australian population than a Chinese population. Obes Res Clin Pract. 2018; 12(5):438-44.

22. Qiu Y, Zhao Q, Gu Y, Wang N, Yu Y, Wang R, et al. Association of Metabolic Syndrome and its Components with decreased estimated glomerular filtration rate in adults. Ann Nutr Metab. 2019;75:168-78.

23. Levey AS, Stevens LA, Schmid CH, Zhang YL, Castro AR, Feldman HI, et al. A new equation to estimate glomerular filtration rate. Ann Intern Med. 2009; 150(9):604-12.

24. Levey AS, Coresh J, Greene T, Stevens LA, Zhang YL, Hendriksen S, et al. Using standardized serum creatinine values in the modification of diet in renal disease study equation for estimating glomerular filtration rate. Ann Intern Med. 2006;145(4):247.

25. Arsenault BJ, Lemieux I, Despres JP, Wareham NJ, Kastelein JJ, Khaw KT, et al. The hypertriglyceridemic-waist phenotype and the risk of coronary artery disease: results from the EPIC-Norfolk prospective population study. CMAJ. 2010;182(13):1427-32.

26. American Diabetes Association. Executive summary: Standards of medical care in diabetes--2014. Diabetes Care. 2014;37(Suppl 1):S5-13.

27. Chobanian AV, Bakris GL, Black HR, Cushman WC, Green LA, Izzo JJ, et al. Seventh report of the joint National Committee on prevention, detection, evaluation, and treatment of high blood pressure. Hypertension. 2003;42(6): 1206-52.

28. He S, Zheng Y, Shu Y, He J, Wang Y, Chen X. Hypertriglyceridemic waist might be an alternative to metabolic syndrome for predicting future diabetes mellitus. PLoS One. 2013:8(9):e73292.

29. Coresh J, Turin TC, Matsushita K, Sang Y, Ballew SH, Appel L, et al. Decline in estimated glomerular filtration rate and subsequent risk of end-stage renal disease and mortality. JAMA. 2014;311(24):2518-31.

30. Despres JP, Lemieux I, Bergeron J, Pibarot P, Mathieu P, Larose E, et al. Abdominal obesity and the metabolic syndrome: contribution to global cardiometabolic risk. Arterioscler Thromb Vasc Biol. 2008;28(6):1039-49.

31. Weinberg JM. Lipotoxicity. Kidney Int. 2006;70(9):1560-6.

32. Kwakernaak AJ, Zelle DM, Bakker SJ, Navis G. Central body fat distribution associates with unfavorable renal hemodynamics independent of body mass index. J Am Soc Nephrol. 2013;24(6):987-94.

33. Thomas R, Kanso A, Sedor JR. Chronic kidney disease and its complications. Prim Care. 2008;35(2):329-44.

34. Chan CM. Hyperlipidaemia in chronic kidney disease. Ann Acad Med Singap. 2005;34(1):31-5.

\section{Publisher's Note}

Springer Nature remains neutral with regard to jurisdictional claims in published maps and institutional affiliations.
Ready to submit your research? Choose BMC and benefit from:

- fast, convenient online submission

- thorough peer review by experienced researchers in your field

- rapid publication on acceptance

- support for research data, including large and complex data types

- gold Open Access which fosters wider collaboration and increased citations

- maximum visibility for your research: over $100 \mathrm{M}$ website views per year

At BMC, research is always in progress.

Learn more biomedcentral.com/submissions 